\title{
Facet Joint Violation by Thoracolumbar Percutaneous Pedicle Screw and Its Effect on Progression of Facet Joint Osteoarthritis
}

\author{
Takeshi Sasagawa \\ Department of Orthopedics Surgery, Toyama Prefectural Central Hospital, Toyama, Japan
}

\section{Study Design: A retrospective study.}

Purpose: This study aimed to investigate the rate and associated factors of facet violation (FV) in percutaneous pedicle screws (PPS) from the thoracic to the lumbar spine and the effect of FV on facet osteoarthritis (OA) progression.

Overview of Literature: Some reports claim PPS has a higher FV rate than conventional open surgery. However, previous reports of FV in PPS were limited to the lumbar spine; only a few reports included the thoracic spine.

Methods: The present study includes 1,028 PPS inserted from T4 to S1 in 218 patients. The rate of FV and facet OA progression after FV were assessed using computed tomography (CT) scans conducted postoperatively at 1 week and 6 months or more. To identify factors associated with $\mathrm{FV}$ or facet $\mathrm{OA}$ progression after $\mathrm{FV}$, a multivariate logistic regression analysis was conducted. To investigate whether $\mathrm{FV}$ caused facet $\mathrm{OA}$ progression, we compared $\mathrm{OA}$ progression between patients with $\mathrm{FV}$ and matched controls.

Results: FV was observed in $68(6.6 \%)$ of the 1,028 facets, and the thoracic spine was identified as an independent factor associated with FV. OA progression was detected in $48.2 \%$ of the cases with FV via CT scans conducted postoperatively at a mean duration of 22.6 months. The time between CT scans was identified as an independent factor for facet OA progression after FV. The rate of OA progression in patients with $\mathrm{FV}$ was significantly greater than that of the controls.

Conclusions: FV was observed in $6.6 \%$ of the patients, and the thoracic spine was identified as an independent factor associated with FV. OA progression of a violated facet occurs over time. FV is considered a complication leading to facet $\mathrm{OA}$ progression.

Keywords: Zygapophyseal joint; Thoracic vertebrae; Lumbar vertebrae; Pedicle screw; Osteoarthritis

\section{Introduction}

Spinal stabilization using percutaneous pedicle screws (PPS) has many advantages, including less blood loss and muscle invasiveness compared with conventional open surgery. It is a widely accepted method for the manage- ment of a variety of spinal conditions requiring stabilization from the thoracic to the lumbar spine [1-4]. In contrast, there are some reports that the facet violation (FV) rate of PPS is higher than that of the conventional open surgery [5,6]. Clinically, superior FV is thought to contribute to accelerated adjacent segment disease

Received Jun 3, 2021; Revised Jul 5, 2021; Accepted Jul 11, 2021

Corresponding author: Takeshi Sasagawa

Department of Orthopedics Surgery, Toyama Prefectural Central Hospital, 2-2-78 Nishinagae, Toyama city, Toyama prefecture, 9308550 , Japan

Tel: +81-76-424-1531, Fax: +81-76-422-0667, E-mail: ortho_spine@tch.pref.toyama.jp 
(ASD) after instrumented spinal fusion [7,8]. Although the natural history of the adjacent disc and the increased biomechanical stress placed on the segments adjacent to the fusion construct are inevitable, the iatrogenic disruption of the adjacent facet joint can contribute to ASD and is potentially avoidable. The rate of ASD varies widely in literature, from $5.2 \%$ to $100 \%$ [7]. The rate of symptomatic ASD is lower, however, and ranges from $12.2 \%$ to $18.5 \%$ in patients with transpedicular instrumentation [7]. This highlights the importance of avoiding adjacent FV during instrumented posterior fusions, and thus understanding the nuances of FV in PPS is important. However, previous reports that referred to FV in PPS were limited to the lumbar spine [5,6,9-13], and only a few reports included the thoracic spine [14,15]. Thus, details of FV in PPS including the thoracic spine are still unclear. Furthermore, although FV may be a risk factor for facet osteoarthritis (OA), only a few reports have investigated the occurrence of facet OA after FV $[15,16]$. Therefore, it remains unclear whether FV causes facet OA. This study aimed to investigate the risk and rate of FV in PPS from the thoracic to the lumbar spine, and to determine the effect of FV on facet $\mathrm{OA}$ progression.

\section{Materials and Methods}

\section{Patient}

This study was approved by the medical ethics committee of Toyama Prefectural Central Hospital (approval no., 5932). Informed consent to use all the patient data was obtained from all patients. At Toyama Prefectural Central Hospital (Toyama, Japan), PPS was introduced in February 2013. The present study includes data of 218 patients out of 254 who underwent posterior spinal stabilization using PPS for a variety of spinal conditions requiring stabilization from the thoracic to lumbar spine between February 2013 and October 2020. The 36 patients that were excluded from this study include 34 who had diffuse idiopathic skeletal hyperostosis and two who did not have computed tomography (CT) scans within 1 week postoperatively. There were 115 men and 103 women with a mean age of $62.3 \pm 15.6$ years (range, $12-95$ years) a mean body mass index (BMI) of $23.1 \pm 3.9 \mathrm{~kg} / \mathrm{m}^{2}$ (range, $13.7-$ $38.2 \mathrm{~kg} / \mathrm{m}^{2}$ ). A total of 1,161 PPS were inserted from T4 to S1 across the 218 patients; 133 facet joints were excluded because of an associated facetectomy. Of the remaining
1,028 screws, 516 were inserted on the right side and 512 on the left side. The levels were classified into three regions [15]: the thoracic spine (T4-T11, $\mathrm{n}=226$ ), thoracolumbar spine (T12-L1, $\mathrm{n}=179$ ), and lumbosacral spine (L2-S1, $\mathrm{n}=623$ ). Among the 218 patients, 110 presented with degenerative disease ( $\mathrm{n}=337$ PPS), 68 with trauma ( $\mathrm{n}=416$ PPS), 21 with a tumor ( $\mathrm{n}=138$ PPS), and 19 with infection ( $\mathrm{n}=137$ PPS). All PPS insertions were conducted by a single surgeon using fluoroscopy. The entry point was at 2 or 10 oclock, along the right or left side of the pedicle, respectively, using a posteroanterior view technique with a lateral-to-medial trajectory [14].

$\mathrm{FV}$ was assessed using CT scans conducted postoperatively within 1 week. FV was classified as a craniocaudal violation, a mediolateral violation, or a screw head violation [15]. Craniocaudal screw violations were further classified as extraarticular (grade 1), intraarticular (involving the caudal third of the facet joint; grade 2), or transarticular (grade 3) (Fig. 1). Mediolateral screw violations were further classified as extraarticular (grade 1), intraarticular (involving the lateral third of the facet joint; grade 2), or transarticular (grade 3) (Fig. 1). The extent of facet depression caused by the screw head was further classified

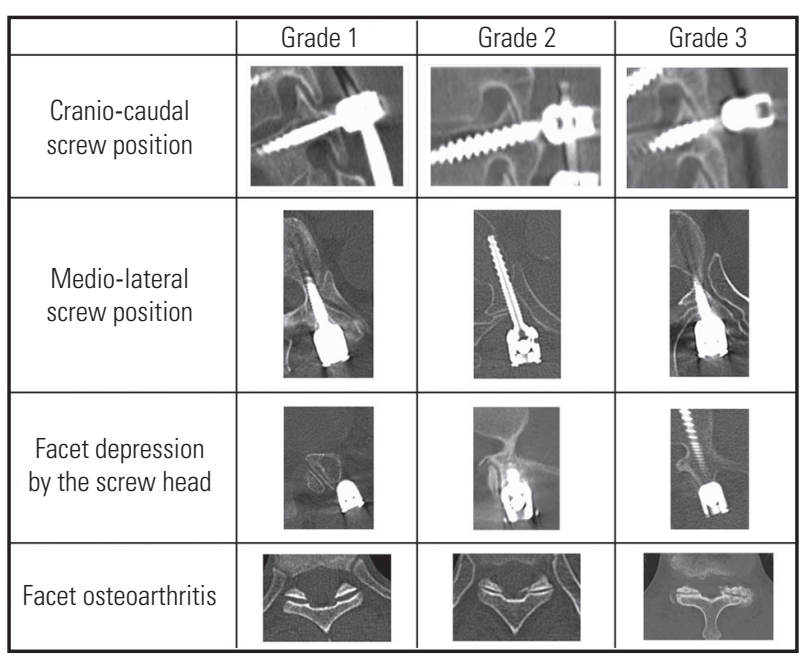

Fig. 1. Grading of the cranio-caudal screw position: grade 1, extraarticular; grade 2, intraarticular involving the caudal third of the facet joint; grade 3, trans-articular. Grading of the medio-lateral screw position: grade 1, extraarticular; grade 2, intraarticular involving the lateral third of the facet joint; grade 3, trans-articular. Grading of the extent of facet depression caused by the screw head: grade 1, no depression; grade 2 , depression $<1 / 3$ of the facet joint; grade 3 , depression $>1 / 3$ of the facet joint. Grading of facet osteoarthritis: grade 1, absent; grade 2, minor osteoarthritis characterized by cartilage thinning, subchondral sclerosis and/or presence of intraarticular gas; grade 3: severe osteoarthritis if the joint space was very narrow and osteophytes present. 
as: no depression (grade 1), depression $<1 / 3$ (grade 2), or depression $>1 / 3$ of the facet joint (grade 3) (Fig. 1) [15].

\section{Rate and associated factors of facet violation}

The rate and type of FV were investigated at each level of instrumentation. We divided the screws into the FV and non-FV groups. We compared the two groups for sex, age, BMI, type of disease, level of instrumentation, laterality, and PPS diameter and length. To understand the influence of the learning curve on FV, the consecutive surgeries were numbered chronologically from 1 to 254 , which included the 36 patients excluded from the study. To identify the factors associated with FV, a multivariate logistic regression analysis was conducted wherein the presence or absence of FV was used as a dependent variable, and items that were significantly different on univariate analysis were independent variables.

\section{Occurrence and associated factors of progression of facet osteoarthritis after facet violation}

The occurrence and progression of facet OA were investigated. Of the violated facets, those that had postoperative CT images after 6 months or more were included in this investigation. Facet $\mathrm{OA}$ was classified into three grades (Fig. 1) [15]: absent (grade 1); minor OA (characterized by cartilage thinning, subchondral sclerosis, and/or presence of intraarticular gas; grade 2); or severe OA (very narrow joint space with osteophytes present; grade 3). We defined progression as those wherein the OA grade changed by at least one grade within 1 month or less preoperatively and 6 months or more postoperatively. We divided the $\mathrm{FV}$ facets into the $\mathrm{OA}$ progression and nonprogression groups, comparing the two groups for sex, age, BMI, time between CT scans, type of disease, instrumentation level, range of stabilization, laterality, PPS diameter and length, facet stabilization, and violation grade. The most cranial facet joint of the instrumented spinal segments is defined as the "not stabilized facet", whereas the others are the "stabilized facets". To identify the factors associated with progression, a multivariate logistic regression analysis was conducted wherein the presence or absence of progression was used as a dependent variable, and items which were significantly different on univariate analysis were the independent variables.

\section{Investigation of facet violation as a cause for facet os- teoarthritis progression}

To investigate whether FV caused facet OA progression, we compared OA progression between the FV and nonFV groups. To exclude the influence of items other than $\mathrm{FV}$, we used the nonviolated facets of patients who had violated facets as a control group (non-FV group) and compared the occurrence of facet OA progression between the groups.

\section{Statistical analyses}

All data are expressed as mean \pm standard deviation. Mann-Whitney $U$-test, chi-square test, or Fisher exact test were performed to compare each item, as appropriate. Multivariate logistic regression analysis was used to identify the factors associated with FV and those with progression of facet OA after FV. Differences with $p<0.05$ were considered statistically significant. All statistical analyses were conducted using IBM SPSS Statistics for Windows ver. 22.0 (IBM Corp., Armonk, NY, USA).

\section{Results}

\section{Rate and associated factors of facet violation}

FV was observed in 68 out of the 1,028 facets $(6.6 \%)$ on CT scans conducted postoperatively within 1 week, including 43 screws $(43 / 226,19.0 \%)$ inserted in the thoracic spine, $2(2 / 179,1.1 \%)$ in the thoracolumbar spine, and $23(23 / 623,3.7 \%)$ in the lumbosacral spine. The rate of FV at each vertebra is presented in Table 1. Almost all FVs in the thoracic spine and thoracolumbar junction were craniocaudal and mediolateral. In the lumbosacral spine, screw head violation was more frequent compared with craniocaudal or mediolateral violation. The types and grading of FV at each level are described in Table 2. Tumors occurred at significantly higher rates in the FV group ( $n=68)$ than in the non-FV group $(n=960)$. In contrast, the rate of trauma in the FV group was significantly less compared with that in the non-FV group. The FV group, compared with the non-FV group, had significantly greater proportion of PPS placed in the thoracic spine and significantly less proportion of PPS placed in the thoracolumbar junction and lumbosacral spine. In the FV group, the PPS were significantly smaller in diameter 
Table 1. Rate of FV at each vertebra

\begin{tabular}{|c|c|c|c|c|c|c|c|c|c|c|c|c|c|c|c|}
\hline \multirow{2}{*}{ Variable } & \multicolumn{15}{|c|}{ Level } \\
\hline & T4 & T5 & T6 & T7 & T8 & T9 & T10 & $\mathrm{T} 11$ & $\mathrm{~T} 12$ & L1 & L2 & L3 & L4 & L5 & S1 \\
\hline Total & 14 & 23 & 16 & 18 & 30 & 25 & 34 & 66 & 99 & 80 & 98 & 131 & 201 & 165 & 28 \\
\hline FV & 1 & 6 & 5 & 7 & 11 & 6 & 4 & 3 & 2 & 0 & 1 & 5 & 5 & 11 & 1 \\
\hline Non-FV & 13 & 17 & 11 & 11 & 19 & 19 & 30 & 63 & 97 & 80 & 97 & 126 & 196 & 154 & 27 \\
\hline FV (\%) & 7.1 & 26.1 & 31.3 & 38.9 & 36.7 & 24.0 & 11.8 & 4.5 & 2.0 & 0 & 1.0 & 3.8 & 2.5 & 6.7 & 3.6 \\
\hline
\end{tabular}

FV, facet violation.

Table 2. Types and grading of facet violation

\begin{tabular}{lccc} 
Level & Cranio-caudal violation & Medio-lateral violation & Screw head violation \\
\hline Thoracic spine (T4-11) & $41(3)$ & $38(13)$ & $1(1)$ \\
\hline Thoracolumbar junction (T12-L1) & $2(0)$ & $2(0)$ & 0 \\
\hline Lumbosacral spine (L2-S) & $9(4)$ & $9(7)$ & $15(3)$ \\
\hline Total & $52(7)$ & $49(20)$ & $16(4)$ \\
\hline
\end{tabular}

The values without parentheses describe the total number of FV screws of grade $2+$ grade 3 , and the values given in parentheses are only for grade 3 .

FV, facet violation.

${ }^{\text {a) }}$ Some screws had multiple types of FV.

Table 3. Demographic data and the relationship between FV group and non-FV group

\begin{tabular}{|c|c|c|c|}
\hline Variable & $\begin{array}{l}\text { FV group } \\
(n=68)\end{array}$ & $\begin{array}{c}\text { Non-FV } \\
\text { group }(n=960)\end{array}$ & $p$-value \\
\hline Sex & & & 0.706 \\
\hline Men & 40 & 540 & \\
\hline Women & 28 & 420 & \\
\hline Age (yr) & $64.5 \pm 11.3$ & $60.9 \pm 16.2$ & 0.216 \\
\hline Body mass index $\left(\mathrm{kg} / \mathrm{m}^{2}\right)$ & $22.9 \pm 5.0$ & $22.9 \pm 3.9$ & 0.339 \\
\hline Consecutive no. of surgeries & $128.7 \pm 78.6$ & $123.3 \pm 73.5$ & 0.610 \\
\hline Type of diseases & & & $<0.05$ \\
\hline Degeneration & 16 & 321 & \\
\hline Trauma & 18 & 398 & \\
\hline Infection & 10 & 127 & \\
\hline Tumor & 24 & 114 & \\
\hline Level & & & $<0.05$ \\
\hline Thoracic spine (T4-11) & 43 & 183 & \\
\hline Thoracolumbar junction (T12-L1) & 2 & 177 & \\
\hline Lumbosacral spine (L2-S1) & 23 & 600 & \\
\hline Laterality & & & 0.803 \\
\hline Right & 33 & 483 & \\
\hline Left & 35 & 477 & \\
\hline Screw diameter (mm) & $5.7 \pm 0.7$ & $6.0 \pm 0.5$ & $<0.05$ \\
\hline Screw length (mm) & $41.8 \pm 4.6$ & $44.2 \pm 3.6$ & $<0.05$ \\
\hline
\end{tabular}

Values are presented as number or mean \pm standard deviation.

$\mathrm{FV}$, facet violation. and shorter in length than the non-FV group (Table 3). A multivariate logistic regression analysis was conducted to identify the factors associated with FV. Only the thoracic spine was identified as an independent factor associated with FV (odds ratio, 4.73; 95\% confidence interval, 2.1810.23) (Table 4).

\section{Occurrence and associated factors of progression of facet osteoarthritis after facet violation}

Of the 68 violated facets from 36 patients, 57 facets (83.8\%) from 30 patients had CT images available at 6 months or more after surgery. After excluding one facet because it became osteolytic due to a postoperative tumor, 56 facets were included this investigation. The mean time between CT scans was $22.6 \pm 13.3$ months (range, 6-48 months). Of the 56 facets, OA progressed in 27 facets (48.2\%). The time between CT scans in the OA progression group $(n=27)$ was significantly longer than in the nonprogression group $(n=29)$. Screw length was significantly shorter in the OA progression group than in the nonprogression group (Table 5). A multivariate logistic regression analysis revealed that time between CT scans was the only independent factor associated with progression of facet OA after FV (odds ratio, 1.07; 95\% confidence interval, 1.02-1.12) (Table 6). 
Table 4. Data for each associated factor using binomial logistic regression analysis

\begin{tabular}{lcc} 
Factor & $p$-value & Odds ratio $(95 \%$ confidence interval) \\
Type of disease & 0.32 & $1.50(0.68-3.33)$ \\
\hline Degeneration & 0.75 & $0.87(0.37-2.05)$ \\
\hline Infection & 0.06 & $2.01(0.99-4.09)$ \\
\hline Tumor & & \\
\hline Level & $<0.05$ & $4.73(2.18-10.23)$ \\
\hline Thoracic spine (T4-11) & 0.15 & $0.33(0.07-1.51)$ \\
\hline Thoracolumbar junction (T12-L1) & 0.61 & $0.87(0.50-1.49)$ \\
\hline Screw diameter & 0.31 & $0.96(0.89-1.04)$ \\
\hline Screw length & & -0.182 \\
\hline
\end{tabular}

The category with the most facet violations (trauma for type of disease and lumbosacral spine for level) was used as a reference $\left(R^{2}=0.055\right)$.

\section{Determining whether facet violation causes facet os- teoarthritis progression}

There were 119 nonviolated facets from 30 patients who had violated facets had available CT images at 6 months or more after surgery. After excluding one facet because of facet dislocation due to trauma, 118 facets were used as a control group (non-FV group). Sex, age, BMI, time between CT scans, and type of disease were not different between the FV $(n=56)$ and non-FV groups $(n=118)$. OA progression was observed in $27 / 56$ facets (48.2\%) of the FV group and in $12 / 118$ facets $(10.2 \%)$ of the non-FV group. The rate of OA progression was significantly more frequent in the FV group compared with that in the nonFV group (Table 7).

\section{Discussion}

At present, the rate of FV due to PPS is reported to be between $1.5 \%$ and $58 \%$ [5,6,9-13]. In the present study, the rate of $\mathrm{FV}$ was $6.6 \%$. Although there are some reports concerning the risk factors for FV after PPS, those studies were limited to the lumbar level. Lumbar spine location, L5, high BMI, and large facet angle have been identified as risk factors for FV due to PPS [11-13]. In this study, we included not only the lumbar spine but also the thoracic spine, and we were able to identify the thoracic spine as an independent risk factor associated with FV due to PPS. This high risk in the thoracic spine is thought to be the result of its unique anatomy, such as the coronal facet, the sloped cortex of the transverse process, and the narrow, large sagittally angled pedicle. Previous studies suggested that FV is more likely to occur with a coronal facet in the lumbar spine $[12,13]$. In the thoracic spine, the facet joint is more coronally oriented than in the lumbar spine [17]. The coronal facet blocks the pathway of the PPS. Because of the sloped cortex of the transverse process, inserting a conventional pedicle screw at the thoracic level involves using a burr or rongeur to remove cortical bone over the superior aspect of the pedicle to visualize the cancellous bone [18]. However, the percutaneous approach cannot be achieved with this process. Because the entry point of the thoracic PPS is at a rounded, sloping surface with cortical bone, the starter needle can easily deviate from the entry point and migrate medially to cause FV [18] (Fig. 1). Inserting the pedicle screw at the thoracic level is also technically challenging due to the narrow pedicle [1720]. Because the sagittal angle of the thoracic pedicle is large, it should be inserted along the craniocaudal direction [17,21], which easily can violate the cranial facet joint (Fig. 1). In the present study, the rate of FV at T5-T10 was $>10 \%$, and especially high at T7 and T8, which are levels with a large sagittal angle and narrow pedicle [17,20]. For these reasons, PPS in the thoracic spine are more likely to pass the facet, leading to more frequent craniocaudal and mediolateral FV. The univariate analysis revealed that in the FV group, the PPS were significantly smaller in diameter and shorter in length than the non-FV group. This fact explains how the thoracic spine was a factor associated with FV because the PPS would be smaller and shorter in the thoracic spine than in the lumbar spine. On multivariate logistic regression analysis, only the thoracic spine was identified as an independent factor associated with FV. 
Table 5. Demographic data and the relationship between the OA progression group and non-progression group

\begin{tabular}{|c|c|c|c|}
\hline Variable & OA progression group ( $n=27$ ) & OA non-progression group $(n=29)$ & $p$-value \\
\hline Sex & & & 0.06 \\
\hline Men & 10 & 19 & \\
\hline Women & 17 & 10 & \\
\hline Age (yr) & $64.4 \pm 11.2$ & $67.4 \pm 11.2$ & 0.71 \\
\hline Body mass index $\left(\mathrm{kg} / \mathrm{m}^{2}\right)$ & $22.4 \pm 3.8$ & $21.3 \pm 2.6$ & 0.10 \\
\hline Time between computed tomography (mo) & $28.2 \pm 13.4$ & $17.4 \pm 10.8$ & $<0.05$ \\
\hline Type of disease & & & 0.64 \\
\hline Degeneration & 8 & 5 & \\
\hline Trauma & 6 & 10 & \\
\hline Infection & 8 & 9 & \\
\hline Tumor & 5 & 5 & \\
\hline Level & & & 0.33 \\
\hline Thoracic spine (T4-11) & 18 & 16 & \\
\hline Thoracolumbar junction (T12-L1) & 0 & 2 & \\
\hline Lumbosacral spine (L2-S1) & 9 & 11 & \\
\hline Range of stabilization & & & 0.40 \\
\hline 1 or 2 levels & 7 & 11 & \\
\hline 3 levels or more & 20 & 18 & \\
\hline Laterality & & & 0.40 \\
\hline Right & 14 & 13 & \\
\hline Left & 13 & 16 & \\
\hline Screw diameter (mm) & $5.5 \pm 0.6$ & $5.8 \pm 0.8$ & 0.40 \\
\hline Screw length (mm) & $40.6 \pm 4.4$ & $43.1 \pm 4.6$ & $<0.05$ \\
\hline Facet stabilization & & & 0.78 \\
\hline Not stabilized facet & 3 & 6 & \\
\hline Stabilized facet without anterior support & 21 & 19 & \\
\hline Stabilized facet with anterior support & 3 & 4 & \\
\hline Violation grade & & & 0.09 \\
\hline Grade 2 & 16 & 23 & \\
\hline Grade 3 & 11 & 6 & \\
\hline
\end{tabular}

Values are presented as number or mean \pm standard deviation.

$\mathrm{OA}$, osteoarthritis.

Table 6. Data for each associated factor using binomial logistic regression analysis

\begin{tabular}{lccc} 
Factor & $p$-value & Odds ratio $(95 \%$ confidence interval) & Beta coefficient \\
Time between computed tomography & $<0.05$ & $1.07(1.02-1.12)$ & 0.377 \\
Screw length & 0.09 & $0.89(0.78-1.02)$ & -0.222 \\
\hline$R^{2}-0.213 .22$ &
\end{tabular}

$R^{2}=0.213$.

In the lumbosacral spine, screw head violations were more frequent compared with craniocaudal or mediolateral violations. This may be caused by deep PPS insertion because the depth of the screw head cannot be confirmed directly, and the screw head can violate a sagittally directed lumbar facet joint (Fig. 1). The use of intraopera- 
Table 7. OA grade, progression and the relationship between the FV group and the non-FV group

\begin{tabular}{|c|c|c|c|}
\hline Variable & FV group $(n=56)$ & Non-FV group ( $\mathrm{n}=118)$ & $p$-value \\
\hline Time between computed tomography (mo) & $22.6 \pm 13.3$ & $19.6 \pm 10.1$ & 0.27 \\
\hline OA grade (1 month or less preoperatively) & & & 0.95 \\
\hline 1 & 37 & 84 & \\
\hline 2 & 17 & 33 & \\
\hline 3 & 2 & 1 & \\
\hline OA grade (6 months or more postoperatively) & & & $<0.05$ \\
\hline 1 & 15 & 78 & \\
\hline 2 & 21 & 28 & \\
\hline 3 & 20 & 12 & \\
\hline OA progression & & & $<0.05$ \\
\hline+ & 27 & 12 & \\
\hline - & 29 & 106 & \\
\hline
\end{tabular}

Values are presented as number or mean \pm standard deviation.

$\mathrm{OA}$, osteoarthritis; FV, facet violation.

tive three-dimensional CT computer navigation in the lumbar spine reportedly has a lower FV rate [22], but this requires expensive special equipment that not all facilities are capable of operating. Therefore, using a longer PPS is a simple countermeasure to prevent screw head FV.

In this study, the time between CT scans was identified as an independent factor for the progression of facet $\mathrm{OA}$ after FV. Thus, OA progression from a violated facet may occur over time. To our knowledge, only two reports have investigated the occurrence of facet OA after FV due to PPS, both targeting spinal trauma patients $[15,16]$. These two reports had conflicting findings about whether FV is related to facet OA $[15,16]$. Tromme et al. [15] reported no differences in FV rate between an OA progression versus a nonprogression group. However, the time between CT scans was significantly different between groups, and thus factors other than FV may have contributed to facet OA in that study. Therefore, our study analyzed the nonviolated facets of patients who had violated facets as a control group to exclude other influences. With that approach, we found that OA progression in the FV group was significantly more frequent compared with in the non-FV group. Therefore, we concluded that FV is a complication leading to facet OA progression. Although Proietti et al. [16] reported that FV is related to facet OA, that study had some limitations such as small sample size and short-term follow-up. Our study, which has a larger sample size and longer follow-up supports the finding that
FV is related to facet OA. As the time between CT scans was identified as an independent factor for the progression of facet OA after FV, we conclude that facet OA occurs chronologically even in the nonprogression group. Furthermore, because OA progression was significantly more frequent in the FV group compared with in the non-FV group, FV especially in a nonstabilized cranial adjacent facet or temporally fixed facet should be avoided.

The current study had more PPS inserted by a single surgeon compared with previous studies. It has been reported that the incidence of FV declines with more surgical experience [13]. However, in the present study, the consecutive number of surgeries was not significantly different between the FV and non-FV groups. Thus, FV is a complication that must always be considered, even if the surgeon has become more familiar with PPS insertion.

There are some limitations to the present study. In this study, the FV rate was low (6.6\%), so the sample size of $\mathrm{FV}$ is still small. It is possible that some variables would have been significantly different between the groups if the sample size were larger. For example, although severe FV (grade 3) caused more frequent progression of facet OA than mild FV (grade 2), there was no significant difference in FV grade (Table 5). This study also cannot address ASD caused by FV. In this study, no patient required revision due to ASD caused by FV. This may be the result of a small sample size and short follow-up period because only eight cases (nine facet joints) had FV at the most cranial 
facet joint. Therefore, further studies with a larger sample size and longer follow-up period are needed to verify this. Spinal degeneration, including facet OA, may be affected by overall sagittal alignment, which was not assessed in this study. We were able to identify the independent factors associated with FV or OA progression, but it is not suitable to use those results to predict FV or OA progression because the $R 2$ values of the multivariate model were low. Furthermore, all PPS insertions were conducted by a single surgeon. Therefore, the tendency for the type of FV may be strongly affected by the surgeon's technique. Further studies are necessary to understand the occurrence of FV from PPS and its effect on facet OA.

\section{Conclusions}

FV from thoracolumbar PPS was observed in 68 (6.6\%) out of the 1,028 facets, and the thoracic spine was identified as an independent factor associated with FV. Progression of OA was seen in $48.2 \%$ of violated facets on CT scans conducted postoperatively at a mean duration of 22.6 months. The time between CT scans was identified as an independent factor associated with progression of facet OA after FV. Therefore, OA progression of a violated facet occurs over time. The FV group had significantly greater rate of OA progression than the non-FV group. Therefore, $\mathrm{FV}$ is considered a complication leading to facet OA progression.

\section{Conflict of Interest}

No potential conflict of interest relevant to this article was reported.

\section{ORCID}

Takeshi Sasagawa: https://orcid.org/0000-0002-3849-0178

\section{Author Contributions}

Conception and design of the study, collection and analysis of data, critical revision of the article for important intellectual content: Takeshi Sasagawa.

\section{References}

1. Zairi F, Vielliard MH, Bouras A, Karnoub MA,
Marinho P, Assaker R. Long-segment percutaneous screw fixation for thoraco-lumbar spine metastases: a single center's experience. J Neurosurg Sci 2017;61:365-70.

2. Farah K, Peyriere H, Graillon T, et al. Minimally invasive posterior fixation and anterior debridementfusion for thoracolumbar spondylodiscitis: a 40-case series and review of the literature. Neurochirurgie 2020;66:24-8.

3. Sun XY, Zhang XN, Hai Y. Percutaneous versus traditional and paraspinal posterior open approaches for treatment of thoracolumbar fractures without neurologic deficit: a meta-analysis. Eur Spine J 2017;26:1418-31.

4. Barbagallo GM, Raudino G, Visocchi M, et al. Restoration of thoracolumbar spine stability and alignment in elderly patients using minimally invasive spine surgery (MISS): a safe and feasible option in degenerative and traumatic spine diseases. Acta Neurochir Suppl 2017;124:69-74.

5. Jones-Quaidoo SM, Djurasovic M, Owens RK 2nd, Carreon LY. Superior articulating facet violation: percutaneous versus open techniques. J Neurosurg Spine 2013;18:593-7.

6. Babu R, Park JG, Mehta AI, et al. Comparison of superior-level facet joint violations during open and percutaneous pedicle screw placement. Neurosurgery 2012;71:962-70.

7. Park P, Garton HJ, Gala VC, Hoff JT, McGillicuddy JE. Adjacent segment disease after lumbar or lumbosacral fusion: review of the literature. Spine (Phila Pa 1976) 2004;29:1938-44.

8. Park P, Foley KT. Minimally invasive transforaminal lumbar interbody fusion with reduction of spondylolisthesis: technique and outcomes after a minimum of 2 years' follow-up. Neurosurg Focus 2008;25:E16.

9. Idler C, Rolfe KW, Gorek JE. Accuracy of percutaneous lumbar pedicle screw placement using the oblique or "owl's-eye" view and novel guidance technology. J Neurosurg Spine 2010;13:509-15.

10. Patel RD, Graziano GP, Vanderhave KL, Patel AA, Gerling MC. Facet violation with the placement of percutaneous pedicle screws. Spine (Phila Pa 1976) 2011;36:E1749-52.

11. Park Y, Ha JW, Lee YT, Sung NY. Cranial facet joint violations by percutaneously placed pedicle screws adjacent to a minimally invasive lumbar spinal fu- 
sion. Spine J 2011;11:295-302.

12. Zhao Y, Yuan S, Tian Y, Liu X. Risk factors related to superior facet joint violation during lumbar percutaneous pedicle screw placement in minimally invasive transforaminal lumbar interbody fusion (MIS-TLIF). World Neurosurg 2020;139:e716-23.

13. Xu Z, Tao Y, Li H, Chen G, Li F, Chen Q. Facet angle and its importance on joint violation in percutaneous pedicle screw fixation in lumbar vertebrae: a retrospective study. Medicine (Baltimore) 2018;97:e10943.

14. Tannous O, Jazini E, Weir TB, et al. Facet joint violation during percutaneous pedicle screw placement: a comparison of two techniques. Spine (Phila Pa 1976) 2017;42:1189-94.

15. Tromme A, Charles YP, Schuller S, Walter A, Schaeffer M, Steib JP. Osteoarthritis and spontaneous fusion of facet joints after percutaneous instrumentation in thoracolumbar fractures. Eur Spine J 2019;28:1121-9.

16. Proietti L, Scaramuzzo L, Schiro GR, Sessa S, Tamburrelli FC, Cerulli G. Degenerative facet joint changes in lumbar percutaneous pedicle screw fixation without fusion. Orthop Traumatol Surg Res 2015;101:375-9.

17. Nojiri K, Matsumoto M, Chiba K, Toyama Y. Morphometric analysis of the thoracic and lumbar spine in Japanese on the use of pedicle screws. Surg Radiol Anat 2005;27:123-8.
18. Ishii K, Shiono Y, Funao H, Singh K, Matsumoto M. A novel groove-entry technique for inserting thoracic percutaneous pedicle screws. Clin Spine Surg 2017;30:57-64.

19. Kakarla UK, Little AS, Chang SW, Sonntag VK, Theodore N. Placement of percutaneous thoracic pedicle screws using neuronavigation. World Neurosurg 2010;74:606-10.

20. Heintel TM, Berglehner A, Meffert R. Accuracy of percutaneous pedicle screws for thoracic and lumbar spine fractures: a prospective trial. Eur Spine J 2013;22:495-502.

21. Lehman RA Jr, Polly DW Jr, Kuklo TR, Cunningham B, Kirk KL, Belmont PJ Jr. Straight-forward versus anatomic trajectory technique of thoracic pedicle screw fixation: a biomechanical analysis. Spine (Phila Pa 1976) 2003;28:2058-65.

22. Ohba T, Ebata S, Fujita K, Sato H, Haro H. Percutaneous pedicle screw placements: accuracy and rates of cranial facet joint violation using conventional fluoroscopy compared with intraoperative threedimensional computed tomography computer navigation. Eur Spine J 2016;25:1775-80. 